\title{
Chaos Theory: Optimizing Critical Illness Outcomes through the Family Experience A Theoretical Review
}

\author{
Kirby P. Mayer ${ }^{1 \star}$, Samantha A. Mancuso ${ }^{2}$ and Dana M. Howell ${ }^{3}$ \\ ${ }^{1}$ Physical Therapist/Research Assistant, Rehabilitation Sciences \\ Doctoral Program, College Health Sciences, University of \\ Kentucky,900 S. Limestone, CTW Lexington, KY 40536-0200. \\ ${ }^{*}$ Corresponding author \\ Kirby P. Mayer, Physical Therapist/Research Assistant, Rehabilitation \\ Sciences Doctoral Program, College Health Sciences, University of \\ ${ }^{2}$ Adult Gerontology Acute Care Nurse Practitioner, University of \\ Kentucky 740 S LIMESTONE L543 KY CLINIC LEXINGTON, KY \\ Kentucky, 900 S. Limestone, CTW Lexington, KY 40536-0200; E-Mail: \\ kpmaye2@uky.edu.
} 40536-0284.

${ }^{3}$ Occupational Science and Occupational Therapy at Eastern Submitted: 19 June 2017; Accepted: 26 June 2017; Published: 04 July 2017 Kentucky University,USA.

KeyWords: intensive-care unit survivor; family involvement in critical care; chaos theory; interdisciplinary; family-centered care

\section{Introduction}

An estimated six to eight million patients are admitted to the intensive care unit (ICU) in the United States every year [1, 2]. It is possible that new and innovative medical therapies have provided patients with treatment options that enhance survival of a critical illness and allow them go home with more medical support than previously available in earlier years. This may explain why there are now millions of ICU survivors are a result of declining mortality rates $(8-19 \%)$ even though there are increased ICU admissions [3, 4]. This also indicates a change in direction of the attitudes and beliefs of the family unit today and their desire for the family member to return home after critical illness instead of moving to a tertiary facility for care. However, survivorship is not without its negative consequences especially financially. In 2005, the cost of critical illness in the ICU was estimated at $\$ 81.7$ billion [5]. This in combination with frequent re-admission rates and significantly higher utilization of post ICU healthcare resources has contributed to a significant financial burden for ICU survivors and their families $[2,6]$.

In addition to the financial burden, patients are at more risk of developing extensive disability due to the complexity of treating critical illness. Recent research on critical care demonstrates that patients suffer significant physical and cognitive impairments during and following ICU admission which may impact activities of daily living that promote independence [6-8]. Cognitive disability, impaired mobility, and reduced functional status collectively encumber ICU survivors from restoring their preadmission overall health. This may lead to dependence of the ICU survivor on the family unit that is likely to be time consuming considering the assistance needed with daily tasks and increased stress placed on the system as a whole. Thus, critical care places a considerable burden on the patient's family and society.

Researchers are actively looking for solutions to reduce the impact of critical care on the family unit, including the patient and other

members. The perceptions and consequences of having a loved one admitted to the ICU have been well researched. Family members of patients in the ICU have high rates of anxiety, depression, and Post-Traumatic Stress Disorder (PTSD) [9-12]. Observing a lovedone receive care in the ICU can be detrimental to the physical, mental, and emotional well-being of the family, hindering their ability to support and provide care for their loved one. Families are extensively relied on in the ICU not only to support the patient, but also make decisions in cases when the patient is deemed incompetent such as when the patient has an altered mental state related to sedation, delirium, or pathological process. For that reason, the perspective and satisfaction of families in the ICU are relevant to the patient. Thus, the family experience and perception of the ICU is pertinent to the culture of healthcare; the family's physical and cognitive state have the potential to affect patient outcomes [13, 14].

While there is significant evidence about the perceptions of the family in the ICU, evidence evaluating the family's potential to reduce hospital readmission rates, decrease emergency services utilization, and improve societal productivity post critical illness is limited. The purpose of this review is to establish and accentuate the role of the family during and following their loved one's admission to the ICU. For this review, the family is defined as any individual(s) providing support or making decision during the ICU stay and/or any person providing direct care or financial support. The chaos theory will furnish a theoretical framework to foster an overarching model of critical illness focused on family interventions. The chaos theory elucidates the dynamicity of critical illness during ICU admission helping to define the chaotic systems that affect the family experience. The chaos theory is not an explanation of random events, but rather how chaotic systems can be managed to influence outcomes. In theory, an educated and active family would reduce hospital re-admission rates and healthcare associated costs, therefore, decreasing the economic burden on society. Furthermore, an involved and capable family can help to reduce patient disability and promote recovery following critical illness. 
Chaos Theory in Critical Illness

Although chaos theory is derived in math and science, it is now being applied to healthcare and patient outcomes $[15,16]$, and is appropriate to help explain the interaction between the family, the critically ill patient, and the ICU. Small differences in the initial Chaos theory helps to further explain how disorder, turbulence, instability and processes may appear random, but are seemingly not random [15]. It has been noted, that a dynamic, non-linear system that exhibits chaotic behavior possesses a feedback mechanism (either negative or positive) that allows it to evolve incrementally over time; this is dependent on initial circumstances [15]. The initial circumstances of critical illness include the patient's presentation in relation to severity of clinical condition, expected prognosis, and ability to recover. Other influences include how information is delivered to the family during times of stress and what other additional stressors such as the ICU environment, inconsistency of providers, availability of psychosocial support and financial resources are needed (See Figure 1). When behavior over time is appropriately adapted to achieve a specific outcome, constraints or "strange attractors" are evident and can be described or analyzed [17]. The randomness of strange attractors give rise to fractals the qualities or relationships that otherwise appear random [18]. In regards to the role of families during a patient's critical illness, these relationships are the focus of reducing uncertainty; this can be achieved through stressor reduction which includes but is not limited to the immediate crisis period as well as preparation for recovery (if appropriate).

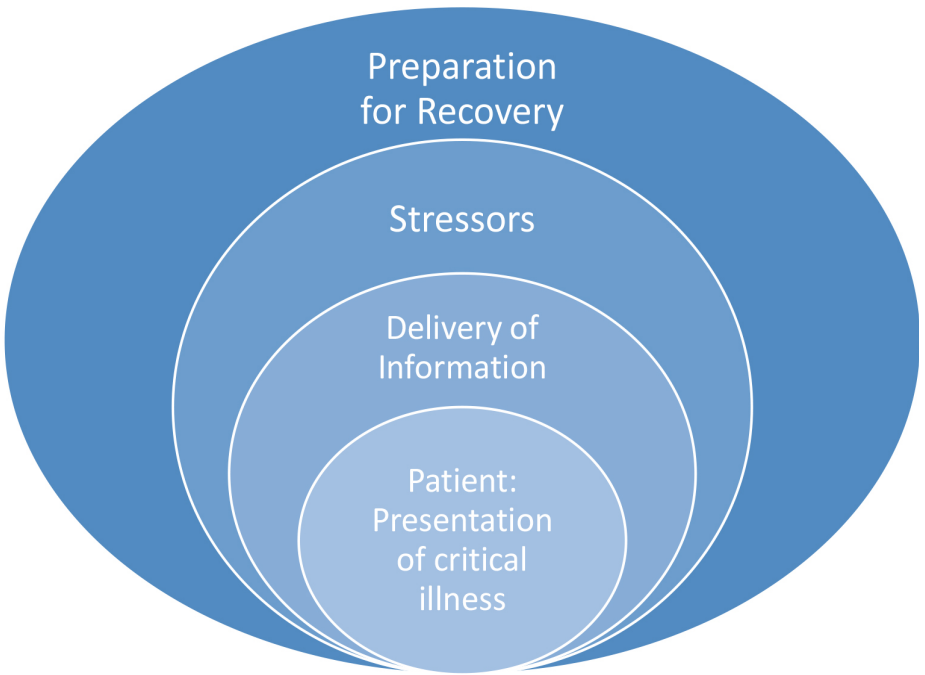

Figure 1: Spheres of influence on the chaotic systems a family experiences when a loved one is admitted to the ICU starts with patient presentation at the time critical illness starts, delivery of information, stressors that impact the critical illness phase, and preparation for recovery. Patient Presentation of critical illness includes the initial conditions present when the patient is first admitted. This would include the diagnosis, expected treatment, acuity, and prognosis of the patient. The next sphere is the delivery of information from the healthcare system including the availability of providers in respect to time and frequency of updated information in addition to multiple disciplines involved in the care of a critically ill loved one. The third sphere is encompassing of all the stressors a family might feel. This includes uncertainty of the future and alterations or variations in the environment with respect to light, noise, and routines. It is strongly impacted by psychosocial support available both medically and within the community. Significant stressors also include financial burden. The fourth and final sphere is the preparation for recovery which includes education and engagement of the family as well as physical, social, financial resources available to the patient once discharged from the hospital. conditions can often yield widely diverging outcomes [15, 16].

This review utilizes an interdisciplinary approach to familycentered care during and following admission to the ICU. The use of chaos theory can be applied to emphasize methodology aimed at reducing familial stress and anxiety by controlling the chaotic systems to enhance their experience. By evaluating the family experience during critical illness, interventions can be developed and optimized in order to improve patient clinical outcomes.

\section{The Impact of Critical Illness on Family: Stress in the ICU} Environmental Factors

When a loved one is admitted to the intensive care unit family members experience significant mental, emotional, and physical stress. A daunting and overwhelming ICU environment creates additional anxiety with sources including complex medical equipment, frequent alarms and noise, bright lights, as well as many medical professionals entering and exiting the room with little explanation [19-21]. This can be frustrating for families to stay up to date on the latest information and focus on their loved one's state of being. Unexpected changes in the patient's medical condition such as clinical deterioration can also add to the unfamiliarity of the ICU setting. Families who are present during times of patient agitation or uncontrolled pain may further potentiate the stress of the environment; these have been identified as a concern and source of stress [20]. Thus, the stress that a family often times experiences evolves from situations in which family members feel disorganized and helpless [11].

\section{Psychosocial Factors}

A family member's ability to cope can negatively impact their stress level, placing them at high risk for depression and anxiety conditions $[11,12,22]$. Stress contributing to a lack of sleep, abnormal dietary habits, and reduced physical activity can alter the mental, emotional, and physical health of the family unit. Emotions are often intensified by uncertainty, unfamiliarity, inadequate communication by providers, and unmet psychological, physiological and social needs [10, 11, 23, 24]. Family members often report feelings of shock, guilt, fear, worry and anxiety over the critical illness their family member experiences $[10,11,23$, 24]. These symptoms help to explain why families of patients with critical illness develop post-traumatic stress disorder (PTSD), a common diagnosis of relatives with family members in the ICU [22].

\section{Family Expectations in Recovery}

Stress may continue to persist following discharge when the patient and family attempt to rectify life at home. Restoring life at home can provide many obstacles from financial burden to functional disability; family members often accept foreign roles as primary care-givers or as fiscal providers. These new roles aggravate stress especially if a family member becomes the primary caregiver and has little to no training, prior education, or experience [25]. Assisting an individual with significant disability can be both physically and cognitively taxing, especially for the family who provide primary care. Additionally, the dedication and time spent by caregivers can be up to 60 hours of direct care each week following a patient's critical illness [25]. This may negatively affect the ability of the family member to carry a full-time job and result in unintended unemployment. This is commonly referred to as caregiver role strain and can have a profound effect on the family as a whole. Thus, the impact critical illness has on families following a member's discharge from the ICU can have serious implications. 
Financial Burden on Family

Critical illness can cause significant financial strain on the patient and family unit. In 2011, the mean hospital charge was 2.5 times higher for discharges with ICU services than for those admitted without ICU services ( $\$ 61,800$ vs $\$ 25,200$, respectively) [26]. This indicates a significant difference in the financial responsibility of the patient and family following critical illness especially if the patient is unable to return to work due to functional loss. The combination of the patient's ability to return to work, household income, and medical health insurance coverage may result in a large financial responsibility.

Financial burden may further be conveyed by re-hospitalization; there is a significant increase in probability of re-admission following ICU stay [2, 6, 7]. According to Hua et al., 16\% of ICU survivors are re-admitted to the hospital within 30 days of initial discharge and an additional $19 \%$ are re-hospitalized within 6 months [2]. furthermore, this contributes to the lost productivity and potential earnings of not just the patient but the family member who must provide care for them. Examples of sources of lost productivity include a period of unpaid leave from work or unexpected loss of employment due to responsibilities at home. This contributes to the family's ability to have access to care and resources.

Thus, the stress of caring for a loved one during and following admission to the ICU is complicated and affected by many dynamic variables. Utilizing the chaos theory, targeted interventions can be implemented to maximize the family experience and improve patient outcomes (See Figure 2).
Crisis Period

The chaos theory explains that the underlying function or malfunction of the dynamic systems along with their relationship can produce a predictable outcome. For this review, the sensitive initial conditions can be elucidated as the crisis period when the family first learns of the severity of the patient's primary medical condition and prognosis. The defined preliminary condition of the family receiving this information can be complex and dynamic. It is dependent on numerous variables including the acuity of the patient, how clinical information is delivered, and expected outcomes. Other influences include psychosocial support available, the physical environment and timing of crucial conversations. Early comprehension of the prognosis has a profound effect on the state of the family. Ineffective communication and delivery of the initial condition can elicit compelling responses. For examples, a family member receiving information from a healthcare provider compared to second-hand information may provoke significantly different responses.

\section{Chaos theory in the ICU}

In context to the family, three main chaotic systems are present during and following ICU admission: the patient's evolving medical status/condition, fluctuating healthcare providers, and the instability in daily schedules (See Table 1). Secondary systems also affect the family including, but not limited to spiritual beliefs, socioeconomic status, community support, prior knowledge/ exposure to healthcare, and health of the individual family member. Over time, each chaotic system affects and influences the other. The systems evolve as the patient's medical condition progresses and the family responses change accordingly.

Table 1: Three main non-linear dynamic (chaotic) systems influencing the family during admission to the ICU. Table 1 elucidates the dynamicity of critical illness and how critical illness in the ICU affects the family.

\begin{tabular}{|c|c|c|}
\hline CHAOTIC SYSTEMS & DYNAMICS & INFLUENCE ON FAMILY \\
\hline $\begin{array}{l}\text { Patient's Medical Status \& } \\
\text { Prognosis }\end{array}$ & $\begin{array}{l}\text { - Status and prognosis can vary by the hour } \\
\text { - Improvements or deteriorations due to or } \\
\text { perceivably unrelated to medical treatment } \\
\text { - Inconsistency or lack of improvement }\end{array}$ & $\begin{array}{l}\text { - Relative consistency with no improvement leads to } \\
\text { uncertainty and anxiety } \\
\text { - } \quad \text { Deteriorations exacerbate stress and feelings of helpless } \\
\text { Improvements can elicit new issues leading to } \\
\text { unfamiliarity (need for caregiving). }\end{array}$ \\
\hline $\begin{array}{l}\text { Patient's Healthcare } \\
\text { Providers }\end{array}$ & 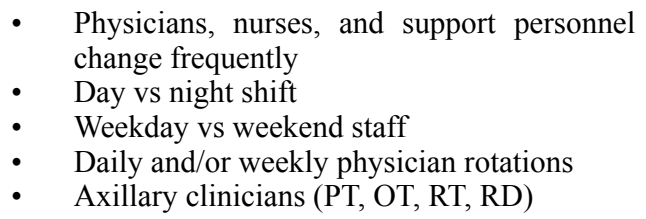 & $\begin{array}{l}\text { - Reduced continuity of care leads to uncertainty, } \\
\text { unfamiliarity, and anxiety } \\
\text { - Inconsistency can reduce trust and communication } \\
\text { Changes in medical status lead to new/more clinicians } \\
\text { increasing complexity of care }\end{array}$ \\
\hline ICU Schedule & $\begin{array}{l}\text { - Planned or unplanned procedures, tests, and } \\
\text { secondary care } \\
\text { Availability of healthcare provider (surgeon, } \\
\text { nurse, PT, OT) } \\
\text { - Availability of resources (OR schedule) } \\
\text { Variability in secondary outcomes (delivery } \\
\text { of breakfast or lunch, bathing at different } \\
\text { times). }\end{array}$ & $\begin{array}{l}\text { - Higher severity or acuity can delay or rush interventions } \\
\text { exacerbating disorder } \\
\text { - Deteriorations in status may emphasize unplanned } \\
\text { procedures and uncertainty } \\
\text { - Complexity of schedule can reduce communication and } \\
\text { involvement of family } \\
\text { - Stress is intensified by new treatment and environments } \\
\text { (OR, imaging, CRRT, intubation) }\end{array}$ \\
\hline
\end{tabular}

Abbreviations: PT (physical therapist), OT (occupational therapist), RT (respiratory therapist), RD (registered dietician), OR (operating room), CRRT (continuous renal replacement therapy)

During admission to the ICU, the main chaotic systems rapidly transform: a critically ill patient's medical status can fluctuate hourly, healthcare providers change frequently, and the ICU schedules are frequently revised. Each system has a relationship to each other. A deterioration in medical status will alter the daily schedule and likely increase the number of healthcare specialists involved in the patient's care. A severe decline in respiratory status may lead to the introduction of a pulmonologist and respiratory 
therapist while altering the schedule to include imperative diagnostic testing. Collectively, the rapid and chaotic changes can have a significant impact on the family's emotional, mental, and physical well-being (See Figure 2).

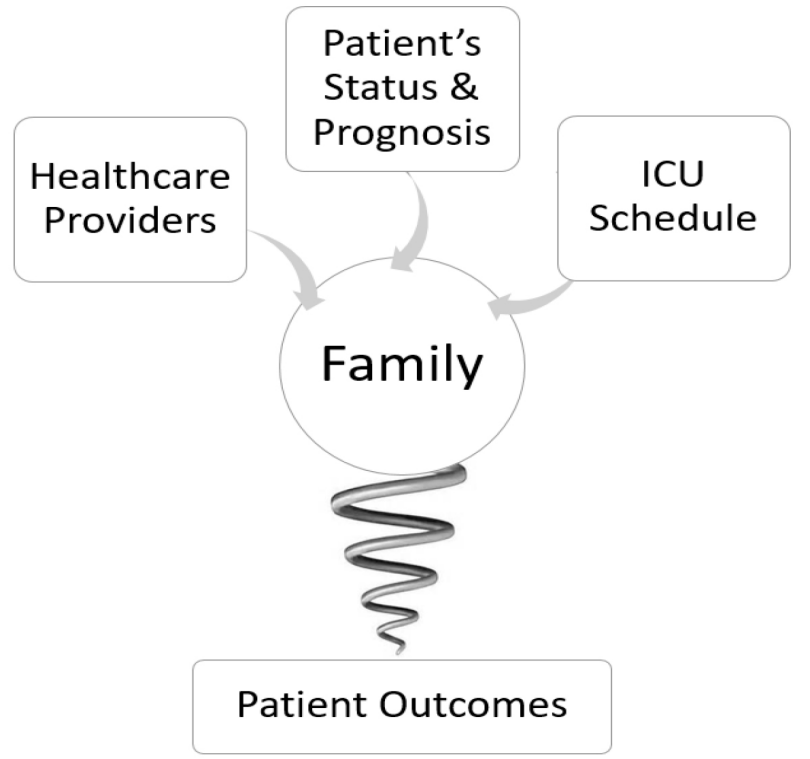

Figure 2: A simplistic visual model of depicting the three main systems (secondary systems not illustrated) that influence the family during a loved one's admission to the ICU. The model reveals how each system influence the family which can lead to positive or negative patient outcomes. The model does not show how the initial condition or how time affects the chaotic systems.

\section{Chaos theory during recovery}

At home, the systems remain dynamic and non-linear, but changes typically occur at a slower rate. Patient's response to treatment should have a linear predicted trajectory, but recovery after critical illness tends to occurs non-linearly. Recovery is influenced by the chaotic systems leading to significant variability, sensitivity and complexity [27]. As noted previously, the unpredictable nature of recovery leads to uncertainty and feelings of helplessness within the family. The recovery phase of critical illness has substantial variability including the possibility of re-hospitalization, limitations to rehabilitation and poor restoration of quality of life prior to the patient's critical illness.

Proposed Interventions to Mitigate the Chaotic Systems of Critical Illness

Controlling the Initial Crisis Period

Chaos theory highlights that the dynamic systems have a sensitive dependency on the initial conditions. Controlling the variability in the crisis period could have a profound effect. In practice, family members are typically informed of their loved one's initial prognosis by a provider (physician, nurse practitioner, or physician assistant). Although the provider may feel comfortable in the hospital, the family typically experiences emotions of helplessness, shock, and disbelief. For the provider, timing and delivery of the patient's medical prognosis can be chaotic in nature, especially if interrupted by clinical deterioration of other patients. Azoulay et al. state that a significant percentage of first meetings with a medical representation last less than ten minutes and frequently do not provide informative brochures [28]. The provider's mood, mannerism, empathy, and professionalism at that precise moment have a powerful influence on how the family comprehends the patient's state.
Thus, the initial crisis period can be managed or controlled to improve the family's' initial interaction. Designating a time and place for delivery of serious information about patient condition may help to control chaos at that point in time. Instead of one provider informing the family, an interdisciplinary team in a designated meeting room should discuss the initial conditions so that multiple perspectives of the patient's condition are presented. Guidance provided by nursing staff and providers is particularly useful to help the family understand how the interdisciplinary care team will control pain, sedation, and agitation during the acute phase of illness [20]. In addition to the patient's medical state and trajectory, the team should assess the family's prior knowledge and experience regarding the ICU. From there, education can be delivered to the family to prepare them for a variety of possible outcomes in addition to orientation of the ICU

During this initial period, emphasis is placed on the patient's immediate physiological needs and acute condition. The family's emotional needs are important but often deferred. Creating interventions that can positively impact this stage of crisis can be difficult but not impossible if small variables are controlled for; small changes can elicit large output [15]. One approach that could significantly decrease chaos in the initial condition would be the introduction of a family-care specialist (FCS), a liaison between the critical care team and the family. Once the family arrives at the hospital, the FCS would organize an interdisciplinary meeting for the family. The designated FCS has potential to decrease the logistical chaos by providing structure in the initial condition and implementing a standardization for routinely delivering information to patient families during times of critical illness. This role was been evaluated noted to positively impact patient length of stay, healthcare costs, communication, and approval of patient care in an ICU $[14,29,30]$. The FCS can foster the relationship between family and healthcare providers maximizing trust and fostering psychosocial support [14]. A structured crisis period that supports the family has the potential to control the initial chaotic systems, positively impacting patient care.

\section{Interventions during ICU Admission}

Communication: In addition to significantly altering the initial condition to optimize family's experience during the crisis period, there are other alternatives to managing the chaotic systems in the ICU. Simplifying the ICU schedule is one way to diminish the family's perception of chaos. Morning "rounds" at a specific time provide the family an opportunity each day to receive updates and express any anxieties or concerns which can positively impact stress. The morning "rounds" also serve as a period for nursing staff to educate the family on the daily schedule such as planned tests, procedures, or rehabilitation sessions. In practice, morning meetings may occur with large variability due to timing and availability of clinicians. A weekly "care conference" to bring together specialists, nurses and the family is likely to enhance communication and promote an overall satisfying family experience [31]. Providing a written schedule for the family as well as summarizing goals and interventions creates structure, reducing uncertainty. A proactive communication strategy and more time allowed for care to be given to the family members has been noted to reduced psychological stress of relatives [20,32].

Education: Additionally, education is crucial to the family response to the chaotic systems in the environment. In compliment to verbal education, clinicians should utilize written and electronic

Volume 2 | Issue 3 | 4 of 6 
media as educational resources. Family members receiving an informative brochure with the standard information about ICU admission were more satisfied with care compared to those family members who did not receive the brochure [10]. In these instances, family members reported lower anxiety levels and greater satisfaction [33]. Early, frequent, and thorough education is a crucial component in potentially reducing re-admission. For example, showing the family member the imaging that identifies a subarachnoid hemorrhage may help the family to understand the severity of the patient's condition. Providing explicit information on the likelihood of complications or potential adverse effects of medications, procedures, and other treatments that may require readmission could also be helpful to families in their mental preparedness. This embodies the forward way of thinking that acute care institutions attempt to implement today with the start of discharge teaching beginning on admission. It is a way to give the family ownership and empowerment of the care their loved one receives. Having the knowledge of not just daily care tasks but resources to help with troubleshooting could possibly eliminate unnecessary ED visits and unplanned readmissions.

Family Presence: Finally, utilization of the family to implement daily patient care is another way chaos within the system may be reduced. Incorporating the family creates a platform to introduce and teach basic care-giving skills that may significantly decrease the stress of care-giving in the recovery stage.

Involving and giving family members responsibility in a simple task has been shown to reduce family anxiety [11]. For example, use of this technique can be as simple as the nursing using teachback methodology with the family for giving a medication. The nurse can instruct the family on appropriate administration, monitoring, and potential adverse effects of the drug. This helps the family to manage and treat certain symptoms once they are home.

Another example of incorporating the family into the patient care during critical illness could be assisting the patient with therapeutic exercises and activities of daily living [34]. Simple involvement in activities such as bathing, feeding, participation in bed mobility, and assisting in rehabilitation activities can significantly enhance the family experience, while simultaneously preparing them for care giving roles during recovery. Certain disciplines such as physical and occupational therapy incorporate on this technique frequently to create a sense of confidence in caregivers and promote active participation in care. By incorporating the family in daily tasks the healthcare team can help the family to focus on the present condition of the patient and develop confidence in the patient's caregiver. Involvement of the family, education, and feedback are all techniques that facilitate patient rehabilitation and promote assurance in the family. In addition, clinicians should encourage the family to allow the patient to perform as much of daily care tasks as possible before assisting to facilitate patient function and independence. For this to be successful, the inclusion of all learning domains (cognitive, affective, and psychomotor) should be used with the family so they understand the significance of their involvement in their loved one's recovery.

Influencing the Recovery Phase

While utilization of the family is important, family resources and community support to decrease role strain in the recovery phase should also be addressed; care giving can be exhausting. Information on what agencies are in the family's community and the services they offer can help decrease stress and promote family wellness. This respite care might have a profound impact on the family experience, preparing them to transition from the hospital to home; they will assume greater roles and responsibility in care.

One intervention to remedy this transition period is the involvement of the FCS as the family begins to adjust this responsibility. The FCS could further serve as a facilitator by assisting the family with resources and advocating for the patient. Tilter et al. state that "making referrals to community resources" and "teaching ways to help families cope" [35] demonstrated incongruences with perceptions of family members following adult critical care. The FCS would ensure appropriate steps are carried out to schedule follow-up visits, obtain necessary home health equipment, ensure physical rehabilitation is assigned, and the family is appropriately managing medications. The FCS would provide communal resources such as support groups for survivors of critical illness and their families, contacts for economic support, and respite care available in the area. The family would also have the power to contact the liaison in situations of ambiguity. The FCS would also provide continuity of care while tracking the patient's medical condition and functional status.

The recovery phase is highly variable for each patient. An educated family has the tools to discern when an emergent situation is occurring compared to when seeking medical care might be necessary. Maintaining interdisciplinary relationships with the patient and family will promote recovery. If the family is educated and supported during the ICU stay, they will be better prepared to for care giving roles, promoting rehabilitation, managing medications, and recognizing adverse reactions or symptoms that would elicit a readmission. This is important because during the recovery phase, the patient and family may have little or no contact with clinicians. This places significant responsibility on the family. Preparation through education and support by the multidisciplinary team prior to the recovery period will help to reduce uncertainty and decrease the fear of the unknown.

\section{Conclusion}

The chaos theory provides a method of anticipating and controlling the perceived randomness of the ICU to enhance functioning [17]. Utilizing this framework, we can begin to understand the complexity of critical illness on the family in three phases: crisis period (the initial conditions), the ICU (hospital) admission and the recovery phase. Maximizing the family's experience during and following ICU admission can have a profound effect on the critical care survivor's outcomes. Furthermore, addressing the immediate and long-term needs of the family can foster a healthy environment for recovery [11]. Theoretically, controlling for the variability within the three phases of critical illness with the use of education, communication, and psychosocial support is likely to minimize chaos within the system. The relationship with the family is placed at the forefront of care along with treating the patient's condition, creating an environment for decreased stress, uncertainty, and fear. Thus, ICU survivor outcomes are likely to be superior by focusing interventions on the family and promoting their care. 


\section{References}

1. Medicine SoCC. Critical Care Statistics (2016).

2. Hua M, Gong MN, Brady J, Wunsch H (2015) Early and Late Unplanned Rehospitalizations for Survivors of Critical Illness (). Critical care medicine 43: 430-438.

3. Young MP, Birkmeyer JD (2000) Potential reduction in mortality rates using an intensivist model to manage intensive care units. Effective clinical practice: ECP 3: 284-289.

4. Bion J, Hall R (2007) Improving the reliability of healthcare systems' responsiveness to the needs of acutely ill patients. Critical care medicine 35: 637-639.

5. Halpern NA, Pastores SM (2010) Critical care medicine in the United States 2000-2005: an analysis of bed numbers, occupancy rates, payer mix, and costs. Critical care medicine 38: $65-71$

6. Herridge MS, Tansey CM, Matte A, et al. (2011) Functional disability 5 years after acute respiratory distress syndrome. The New England journal of medicine 364: 1293-1304.

7. Tansey CM, Louie M, Loeb M, et al. (2007) One-year outcomes and health care utilization in survivors of severe acute respiratory syndrome. Archives of internal medicine 167: 1312-1320.

8. Bienvenu OJ, Colantuoni E, Mendez-Tellez PA, et al. (2012) Depressive symptoms and impaired physical function after acute lung injury: a 2-year longitudinal study. American journal of respiratory and critical care medicine 185: 517-524.

9. Herridge MS, Moss M, Hough CL, et al. (2016) Recovery and outcomes after the acute respiratory distress syndrome (ARDS) in patients and their family caregivers. Intensive care medicine 42: 725-738.

10. Azoulay E, Pochard F, Chevret S, et al. (2003) Family participation in care to the critically ill: opinions of families and staff. Intensive care medicine 29: 1498-1504.

11. Lee LY, Lau YL (2003) Immediate needs of adult family members of adult intensive care patients in Hong Kong. Journal of clinical nursing 12: 490-500.

12. McAdam JL, Puntillo K (2009) Symptoms experienced by family members of patients in intensive care units. American journal of critical care : an official publication, American Association of Critical-Care Nurses 18: 200-209; quiz 210.

13. Davidson JE, Powers K, Hedayat KM, et al. (2007) Clinical practice guidelines for support of the family in the patientcentered intensive care unit: American College of Critical Care Medicine Task Force 2004-2005. Critical care medicine 35: 605-622.

14. Shelton W, Moore CD, Socaris S, Gao J, Dowling J (2010) The effect of a family support intervention on family satisfaction, length-of-stay, and cost of care in the intensive care unit. Critical care medicine 38: 1315-1320.

15. Sharp LF, Priesmeyer HR (1995) Tutorial: chaos theory--a primer for health care. Quality Management In Health Care 3: $71-86$.

16. Holm S (2002) Does chaos theory have major implications for philosophy of medicine? Medical Humanities 28: 78-81.

17. Velde BP, Greer AG, Lynch DC, Escott-Stump S (2002) Chaos theory as a planning tool for community-based educational experiences for health students. Journal of Allied Health 31: 147-152.

18. Haigh C (2002) Using chaos theory: the implications for nursing. Journal of Advanced Nursing 37: 462-469.

19. Wall RJ, Curtis JR, Cooke CR, Engelberg RA (2007) Family satisfaction in the ICU: differences between families of survivors and non survivors. Chest 132: 1425-1433.

20. Schwarzkopf D, Behrend S, Skupin H, et al. (2013) Family satisfaction in the intensive care unit: a quantitative and qualitative analysis. Intensive care medicine 39: 1071-1079.

21. Salandin A, Arnold J, Kornadt O (2011) Noise in an intensive care unit. The Journal of the Acoustical Society of America 130: 3754-3760.

22. Davidson JE, Jones C, Bienvenu OJ (2012) Family response to critical illness: postintensive care syndrome-family. Critical care medicine 40: 618-624.

23. Khalaila R (2014) Meeting the needs of patients' families in intensive care units. Nursing Standard (Royal College Of Nursing (Great Britain): 1987) 28: 37-44.

24. Holden J, Harrison L, Johnson M (2002) Families, nurses and intensive care patients: a review of the literature. Journal of clinical nursing 11: 140-148.

25. Foster M, Chaboyer W (2003) Family carers of ICU survivors: a survey of the burden they experience. Scandinavian Journal of Caring Sciences 17: 205-214.

26. Barrett ML, Smith MW, Elixhauser A, Honigman LS, Pines JM (2011) Utilization of Intensive Care Services, 2011: Statistical Brief \#185. Healthcare Cost and Utilization Project (HCUP) Statistical Briefs 2006.

27. Priesmeyer HR, Sharp LF, Wammack L, Mabrey JD (1996) Chaos theory and clinical pathways: a practical application. Quality Management In Health Care 4: 63-72.

28. Azoulay E, Chevret S, Leleu G, et al. (2000) Half the families of intensive care unit patients experience inadequate communication with physicians. Critical care medicine 28: 3044-3049.

29. Redman C (1997) The role of the neonatal family care specialist. Professional Nurse (London, England) 12: 569571.

30. Nelson DP, Polst G (2008) An interdisciplinary team approach to evidence-based improvement in family-centered care. Critical care nursing quarterly 31: 110-118.

31. Hospital SCs. Reducing Families' Stress in the PICU. 2016; http://www.seattlechildrens.org/about/stories/reducingfamilies-stress-PICU/.

32. Lautrette A, Darmon M, Megarbane B, et al. (2007) A communication strategy and brochure for relatives of patients dying in the ICU. The New England journal of medicine 356: 469-478.

33. Chien WT, Chiu YL, Lam LW, Ip WY (2006) Effects of a needs-based education programme for family carers with a relative in an intensive care unit: a quasi-experimental study. International journal of nursing studies 43: 39-50.

34. Johnson SK, Craft M, Titler M, et al. (1995) Perceived changes in adult family members' roles and responsibilities during critical illness. Image--the journal of nursing scholarship 27: 238-243.

35. Titler MG, Cohen MZ, Craft MJ (1991) Impact of adult critical care hospitalization: perceptions of patients, spouses, children, and nurses. Heart \& lung : the journal of critical care 20: $174-182$.

Copyright: (02017 Kirby P. Mayer et al. This is an open-access article distributed under the terms of the Creative Commons Attribution License, which permits unrestricted use, distribution, and reproduction in any medium, provided the original author and source are credited. 